\title{
171. On a Theorem of Brauer
}

\author{
By Masaru Osima \\ Institute of Mathematics, College of General Education, Osaka University \\ (Comm. by Zyoiti Suetuna, M.J.A., Dec. 12, 1964)
}

The purpose of this paper is to give a simple proof of a theorem of Brauer concerning the principal blocks of characters of finite groups ([4], Theorem 3 , see also [3]).

We refer to Brauer [1], [2]; Brauer-Nesbitt [6]; Osima [8], and Curtis-Reiner [7] as for basic concepts and theorems about the blocks of characters of finite groups.

1. Let $G$ be a group of a finite order and let $p$ be a fixed prime number. We choose the algebraic number field $\Omega$ such that the absolutely irreducible representations of $G$ can be written with coefficients in $\Omega$. Let $\mathfrak{p}$ be a prime ideal divisor of $p$ in $\Omega$ and let $\mathfrak{p}_{\mathfrak{p}}$ be the ring of all $\mathfrak{p}$-integers of $\Omega$, and $\bar{\Omega}$ the residue class field of $\mathfrak{D}_{\mathfrak{p}}(\bmod \mathfrak{p})$. The residue class map of $\mathfrak{D}_{\mathfrak{p}}$ onto $\bar{\Omega}$ will be denoted by an asterisk; $\alpha \rightarrow \alpha^{*}$.

If $M$ is a subset of $G$, we write $|M|$ for the number of elements of $M$. The centralizer of $M$ in $G$ will be denoted by $C_{\theta}(M)$ and the normalizer of $M$ in $G$ by $N_{\theta}(M)$.

The group algebra of $G$ over $\bar{\Omega}$ will be denoted by $\Gamma(G)$ and its center by $Z(G)$. If $M$ is a subset of $G$, we write $[M]$ for the element of $\Gamma(G)$ defined by

$$
[M]=\sum_{m \in M} m .
$$

If $K_{1}, K_{2}, \cdots, K_{m}$ are the conjugate classes of $G$, the elements $\left[K_{1}\right],\left[K_{2}\right], \cdots,\left[K_{m}\right]$ form a basis of $Z(G)$. Let us denote by $\psi_{0}, \psi_{1}, \cdots$, $\psi_{s-1}$ the distinct linear characters of $Z(G)$. The $m$ (absolutely) irreducible characters $\chi_{0}=1, \chi_{1}, \cdots, \chi_{m-1}$ of $G$ are distributed into $s$ blocks $B_{0}, B_{1}, \cdots, B_{s-1}$ for $p$. There exists a one-to-one correspondence between the set of blocks of $G$ and the set of linear characters of $Z(G)$. The block $B_{0}=B_{0}(G)$ of $G$ containing the principal character $\chi_{0}=1$ is called the principal block of $G$.

Since each primitive idempotent of $Z(G)$ is associated with a block of $G$, we shall denote by $\delta_{\tau}$ the primitive idempotent associated with $B_{\tau}$. We then have

$$
\psi_{\tau}\left(\delta_{\sigma}\right)= \begin{cases}1, & \tau=\sigma \\ 0, & \tau \neq \sigma\end{cases}
$$

If we set 


$$
\omega_{i}\left(\left[K_{\infty}\right]\right)=\left|K_{\infty}\right| \chi_{i}\left(u_{\infty}\right) / \chi_{i}(1)
$$

where $u_{\infty}$ is an element in the class $K_{\alpha}$, then the map $\omega_{i}^{*}$ of $Z(G)$ into $\bar{\Omega}$ defined by $\omega_{i}^{*}\left(\left[K_{\alpha}\right]\right)=\left(\omega_{i}\left(\left[K_{a}\right]\right)\right)^{*}$ is a linear character of $Z(. G)$ Two characters $\chi_{i}$ and $\chi_{j}$ belong to the same block, if and only if $\omega_{i}^{*}\left(\left[K_{a}\right]\right)=\omega_{j}^{*}\left(\left[K_{a}\right]\right)$ for all $p$-regular classes $K_{\alpha}$ of $G$, i.e. for classes of $G$ which consist of elements whose order is prime to $p$. For $\chi_{i} \in B_{\tau}$, we have

$$
\psi_{\tau}=\omega_{i}^{*} .
$$

Let $V$ be a set of $p$-regular elements of $G$. We have $|V| \not \equiv 0$ $(\bmod p)([5],[6])$. Hence if we set

$$
\varepsilon_{0}=\left(1 /|V|^{*}\right)[V],
$$

then $\varepsilon_{0} \in Z(G)$ and we have the following

Lemma 1. $\varepsilon_{0}-\delta_{0} \in \operatorname{rad} Z(G)$ where $\operatorname{rad} Z(G)$ denotes the radical of $Z(G)$.

Proof. We have $\chi_{0}([V])=|V|$, and $\chi_{i}([V])=0$ for $\chi_{i} \notin B_{0}(G)$ ([9], Theorem 2). Hence $\omega_{0}^{*}\left(\varepsilon_{0}\right)=1$, and $\omega_{i}^{*}\left(\varepsilon_{0}\right)=0$ for $\chi_{i} \notin B_{0}(G)$. It follows from (1.2) and (1.4) that $\psi_{\tau}\left(\varepsilon_{0}-\delta_{0}\right)=0$ for every $\psi_{\tau}$. This implies that $\varepsilon_{0}-\delta_{0} \in \operatorname{rad} Z(G)$.

If $p$ is prime to the order $|G|$, we have $\varepsilon_{0}=\delta_{0}$. The generalization of Lemma 1 for any block of $G$ and its applications will be shown in another paper.

Let $Q$ be a $p$-subgroup of $G$. We shall say that $u, v \in G$ are $Q$ conjugate, if there exists $\pi \in Q$ such that $v=\pi^{-1} u \pi$. Let $L_{1}, L_{2}, \cdots, L_{t}$ be the Q-conjugacy classes of $G$. The $\left|L_{i}\right|$ is a power of $p$ and $\left|L_{i}\right|=1$, if and only if $L_{i}$ consists of an element in $C_{\theta}(Q)$. Hence $\left|V \cap C_{\theta}(Q)\right|^{*}=|V|^{*}$. Now assume that $Q$ is normal in $G$. If $K_{\alpha} \cap C_{\theta}(G)=\phi$ for $K_{\alpha} \subseteq V$, then $\left[K_{\alpha}\right] \in \operatorname{rad} Z(G)$ ([8], p. 183). Hence if we set

$$
\eta_{0}=\left(1 /|U|^{*}\right)[U]
$$

where $U=V \cap C_{\theta}(Q)$, then we obtain readily

Lemma 2. $\eta_{0}-\delta_{0} \in \operatorname{rad} Z(G)$.

In particular, if $G$ contains a normal $p$-Sylow subgroup $Q$, then we can prove that $\eta_{0}=\delta_{0}$. This will be shown also in another paper.

2. Let $H$ be a subgroup of $G$ and let $h$ be a linear function in $Z(H)$. Then, as in Brauer [2] we define a linear function $h^{\theta}$ in $Z(G)$ by

$$
h^{a}\left(\left[K_{\alpha}\right]\right)=h\left(\left[K_{\alpha} \cap Z(H)\right]\right) .
$$

If $K$ is a subgroup of $G$ such that $H \subseteq K$, then we have $\left(h^{K}\right)^{A}=$ $h^{\theta}$. Denote by $\psi^{\prime}$ the linear character of $Z(H)$ associated with the block $b$, and if $\psi=\left(\psi^{\prime}\right)^{\theta}$ is a linear character of $Z(G)$, then we say 
that $b^{\theta}$ is defined and we set $b^{\theta}=B$ where $B$ is a block of $Z(G)$ associated with $\left(\psi^{\prime}\right)^{G}$.

In the following, if $H$ and $K$ are subgroups of $G$, we shall indicate by $H \subseteq{ }_{0} K$ that $H$ is contained in some conjugate of $K$. Let $Q$ be a $p$-subgroup of $G$ and let $H$ be a subgroup of $G$ such that $Q C_{\theta}(Q) \subseteq H \subseteq N_{\theta}(Q)$. The map $\sigma:\left[K_{\alpha}\right] \rightarrow\left[K_{\alpha} \cap C_{\theta}(Q)\right]$ defines a homomorphism of $Z(G)$ into $Z(H)$ ([1], 7B). As an application, we obtain the following Lemma ([2], 2A).

Lemma 3. Let $Q$ be a p-subgroup of $G$ and let $H$ be a subgroup of $G$ such that $Q C_{G}(Q) \subseteq H \subseteq N_{G}(Q)$. Let b be any block of $H$. Then $b^{\theta}$ is defined. If $B$ is a block of $G$ with the defect group $D$ such that $Q \subseteq_{c} D$, then there exist blocks $b$ of $H$ for which $b^{\theta}=B$.

Now we shall prove the following

Lemma 4. Let $H$ have the same significance as in Lemma 3. Let $b$ be a block of $H$. Then $b^{G}=B_{0}(G)$, if and only if $b=B_{0}(H)$.

Proof. Denote by $\delta_{0}^{\prime}$ the idempotent of $Z(H)$ associated with $B_{0}(H)$. To prove Lemma 4, we need show only that $\sigma\left(\delta_{0}\right)=\delta_{0}^{\prime}$. From our assumption we see that $Q$ is normal in $H$ and that $C_{H}(Q)=C_{\theta}(Q)$. If we set $V \cap H=V^{\prime}$ and $V^{\prime} \cap C_{\theta}(Q)=U^{\prime}$, then it follows from Lemma 2 that $\eta_{0}^{\prime}-\delta_{0}^{\prime} \in \operatorname{rad} Z(H)$ where $\eta_{0}^{\prime}=\left(1 /\left|U^{\prime}\right|^{*}\right)\left[U^{\prime}\right]$. Since $U^{\prime}=V \cap C_{\theta}(Q)$, we have

$$
\sigma\left(\varepsilon_{0}\right)=\left(1 /|V|^{*}\right)\left[V \cap C_{G}(Q)\right]=\left(1 /\left|U^{\prime}\right|^{*}\right)\left[U^{\prime}\right]=\eta_{0}^{\prime}
$$

It follows from Lemma 1 that $\eta_{0}^{\prime}-\sigma\left(\delta_{0}\right) \in \operatorname{rad} Z(H)$ and hence $\sigma\left(\delta_{0}\right)-\delta_{0}^{\prime} \in \operatorname{rad} Z(H)$. Since $\sigma\left(\delta_{0}\right)$ is an idempotent of $Z(H)$, this implies that $\sigma\left(\delta_{0}\right)=\delta_{0}^{\prime}$.

Let $\pi$ be a fixed $p$-element of $G$. If $v$ is a $p$-regular element of $C_{\theta}(\pi)$, we have

$$
\chi_{i}(\pi v)=\sum_{\rho} d_{i \rho}^{\pi} \varphi_{\rho}^{\pi}(v)
$$

for $\chi_{i} \in B$. Here $\varphi_{\rho}^{\pi}$ ranges over the modular irreducible characters of the blocks $b$ of $C_{G}(\pi)$ for which $b^{\theta}=B$. The $d_{i \rho}^{\pi}$ are called the generalized decomposition numbers of $G$. We obtain the following theorem ([4], Corollary 4).

Theorem 1. If $B=B_{0}(G)$, then $\varphi_{\rho}^{\pi}$ in (2.2) ranges over the modular irreducible characters of $B_{0}\left(C_{\theta}(\pi)\right)$.

Proof. Apply Lemma 4 to $G$ and its subgroup $H=C_{A}(\pi)$.

Let $Q$ be a $p$-subgroup of $G$ and let $H$ be a subgroup of $G$ such that $Q C_{G}(Q) \subseteq H$. Then we have

Lemma 5. Let $b$ be a block of $H$ with the defect group $D($ in $H)$. If $Q \subseteq_{c} D$, then $b^{G}=B$ is defined.

Proof. If we apply Lemma 3 to $H$ and its subgroup $\widetilde{G}=Q C_{G}(Q)=$ $Q C_{H}(Q)$, we see that there exist blocks $\widetilde{b}$ of $\widetilde{G}$ for which $\widetilde{b}^{H}=b$. Again, applying Lemma 3 to $G$ and its subgroup $\widetilde{G}, \widetilde{b}^{G}=B$ is defined. 
Hence

$$
b^{G}=\left(\tilde{b}^{B}\right)^{G}=\tilde{b}^{G}=B .
$$

Theorem 2. Let $Q$ be a p-subgroup of $G$ and let $H$ be a subgroup of $G$ such that $Q C_{G}(Q) \leqq H$. Let b be a block of $H$ with the defect group $D$. If $Q \subseteq_{c} D$, then $b^{\theta}=B_{0}(G)$, if and only if $b=B_{0}(H)$.

Proof. Assume first that $b=B_{0}(H)$. Applying Lemma 4 to $H$ and its subgroup $\widetilde{G}=Q C_{G}(Q)$, we have $\left(B_{0}(\widetilde{G})\right)^{B}=B_{0}(H)$. On the other hand, applying Lemma 4 to $G$ and its subgroup $\widetilde{G}$, we have $\left(B_{0}(\widetilde{G})\right)^{\theta}=$ $B_{0}(G)$. Hence $\left(B_{0}(H)\right)^{G}=\left(B_{0}(\widetilde{G})^{H}\right)^{G}=\left(B_{0}(\widetilde{G})\right)^{G}=B_{0}(G)$. Conversely, assume that $b^{G}=B_{0}(G)$. There exist by Lemma 3 blocks $\widetilde{b}$ of $\widetilde{G}$ for which $\widetilde{b}^{H}=b$. Hence $\left(\widetilde{b}^{H}\right)^{G}=\widetilde{b}^{\theta}=B_{0}(G)$. It follows from Lemma 4 that $\widetilde{b}=B_{0}(\widetilde{G})$ and hence we have $b=\left(B_{0}(\widetilde{G})\right)^{H}=B_{0}(H)$.

If we set $Q=D$ in Theorem 2, we obtain ([4], Theorem 3).

\section{References}

[1] R. Brauer: Zur Darstellungstheorie der Gruppen endlicher Ordnung. I. Math. Z., 69, 406-444 (1956).

[2] —-: Zur Darstellungstheorie der Gruppen endlicher Ordnung. II. Math. Z., 72, 25-46 (1959).

[3] - : On blocks of representations of finite groups. Proc. Nat. Acad. Sci., U. S. A., 47, 1888-1890 (1961).

[4] - : Some applications of the theory of blocks of characters of finite groups. I. J. Algebra, 1, 152-167 (1964).

[5] R. Brauer and W. Feit: On the number of irreducible characters of finite groups in a given block. Proc. Nat. Acad. Sci., U. S. A., 45, 361-365 (1959).

[6] R. Brauer and C. Nesbitt: On the modular characters of groups. Ann. of Math., 42, 556-590 (1941).

[7] C. W. Curtis and I. Reiner: Representation Theory of Finite Groups and Associative Algebras. Interscience, New York, London (1962).

[8] M. Osima: Notes on blocks of group characters. Math. J. Okayama Univ., 4, 175-188 (1955).

[9] —-: On some properties of group characters. Proc. Japan Acad., 36, 18-21 (1960). 